\title{
The Extraforaminal Juxtafacet Cyst as a Rare Cause of L5 Radiculopathy: A Case Report
}

\author{
Bernard L. Salmon, MD,* Manuel P. Deprez, MD, PhD, $\dagger$ Achille E. Stevenaert, MD, PhD,* and Didier H.
} Martin, MD, PhD*

From the Departments of *Neurosurgery and $\uparrow$ Neuropathology, State University of Liège, Liège, Belgium.

Study Design. This is a report of a case.

Objective. To document the clinical, radiographic, and histologic characteristics of a lumbar extraforaminal juxtafacet cyst.

Summary of Background Data. Spinal juxtafacet cysts develop most frequently at the dorsal aspect of the zygapophysial joint, sometimes in the posterolateral area of the canal. In one case, they have been described in the foraminal and extraforaminal region.

Methods. Description of the case report.

Result. The authors report one case of a strictly extraforaminal juxtafacet cyst responsible for L5 sciatica. Conclusions. Juxtafacet cysts of the spine represent an infrequent cause of sciatica, usually when they grow in the canal, or more exceptionally when they occupy the foraminal or extraforaminal areas.

Keywords : synovial cyst ; ganglion cyst ; sciatica

Spinal juxtafacet cysts (JFC) may affect any segment of the spine except the sacrum. They usually develop at the dorsal aspect of a degenerated zygapophysial joint and sometimes in the canal, causing spinal cord or root compression. Recently, Kemaloglu et $\mathrm{al}^{1}$ reported a case of a JFC growing in the foraminal and the extraforaminal area. We report a case of lumbar radiculopathy caused by a cyst located strictly in the extraforaminal area.

\section{Case Report}

A 62-year-old man complained of acute inaugural back pain and left sciatica without previous history of trauma or excess of activity.

On admission 2 weeks later, physical examination revealed a severe L5 sensitive and motor deficit with drop foot and a negative straight leg-raising test.

Plain radiograms showed a transitional abnormality with a lombalized SI vertebra, severe disc collapse, and zygapophyseal arthritic changes at the L4-L5 and L5-L6 levels.

A computed tomography (CT) scan of the L5-L6 level demonstrated a prearticular extraforaminal left rounded structure adjacent to the joint that could be responsible for L5 compression. There was no bony erosion.

Magnetic resonance imaging (MRI) appearance was that of a $2 \mathrm{~cm}$ cystic structure, with hypointense T1weighted and hyperintense T2-weighted signal, surrounded by a thin regular rim (Figure 1).

The patient was operated on with an extracanalar approach. Partial drilling of the external part of the joint and of the L6 transverse process allowed the surgeons to reach a gray-rounded structure surrounded by a wellvascularized thin membrane. Careful dissection of the mass from the underlying L5 root and from the joint capsule revealed that the cyst did not communicate with the joint and probably originated from the anterior part of the capsule. En bloc resection was performed without perforating the cyst. Macroscopic examination disclosed a $1 \mathrm{~cm}$ cystic lesion filled with gelatinous content (Figure 2). Histologic features were those of a fibrous pseudocyst, with no epithelial or synovial lining limited by a thick fibrovascular wall and containing fibromyxoid material. Small calcifications were present in the cyst wall, some of them identified as calcium pyrophosphate deposits surrounded by multinucleated giant cells (Figure 3). Postoperative outcome was 
uneventful with complete pain relief.

At the 1-year control examination, the patient presented a complete recovery of motor weakness. The control MRI showed no cyst recurrence.

\section{Discussion}

Juxtafacet cysts are rare conditions with only 3 cases identified in a series of 1500 spinal CT exams. ${ }^{2}$ They have been described at the cervical or thoracic spine and more frequently at the L4-L5 level, associated with an arthritic zygapophyseal joint. ${ }^{3-7}$

They are known to be more frequently located at the dorsal aspect of the joint without causing any root compression. ${ }^{8}$ They also develop into the spinal canal where they become symptomatic because of slow growth or sudden increase of size by bleeding. In our case, there was no evidence of past or recent bleeding, despite a well-vascularized capsule. The reason for the sudden onset of radicular symptoms is unknown. The pathogenesis of the JFC is controversial. As these cysts are more frequently observed at the L4-L5 level, joint mobility seems to play an important role. According to Sabo et al, trauma would be related to $14 \%$ of the cases. ${ }^{6}$ Juxtafacet cysts could result from a synovium herniation through a defective joint capsule, proliferation of pluripotential mesenchymal cells, degeneration of periarticular fibrous tissue, growth of developmental rests of synovial tissue, or tissue metaplasia. ${ }^{3,6,8-11}$

Some recurrent JFCs have been reported. ${ }^{12,13}$ In such cases, one relevant explanation could be the persistence of abnormal joint mobility when a single resection of the cyst is performed. In any event, JFCs are not systematically related to manifest radiologic signs of segmental instability. Moreover, the location of the cyst described in this case report seems more compatible with the hypothesis related to growth of periarticular cells or tissues. Therefore, it is the authors' opinion that the treatment of those pathologies must not systematically be accompanied by arthrodesis.

The radiologic differential diagnosis of extracanalar root compression must mainly include the extraforaminal disc hernia, some benign or malignant tumors originating from the root itself or the surrounding structures. In our patient, as for intraspinal JFC, the superiority of MRI for the radiologic diagnosis has been confirmed.

Many series of patients presenting with an intraspinal JFC have been reported. ${ }^{4-7}$ Their clinical presentations are well described. Up to now, only one case of both foraminal and extraforaminal juxtafacet cyst has been reported by Kemaloglu et al. ${ }^{1}$ In this case, a separation was observed between the articular processes, and it was thought that the cyst was arising from the facet joints and projecting intraforaminally. In our case, the rounded morphology of the cyst and its far lateral position seem to exclude a canalar origin. To our knowledge, this is the first reported case of lumbar root compression by such a strictly extraforaminal JFC, resected by an extracanalar approach.

\section{Key Points}

- Juxtafacet cysts usually develop at the dorsal aspect of the zygapophysial joint.

- They can lead to radicular compression when they develop in the canal or in the foramens.

- Exceptionally, they have an extraforaminal origin and require an extracanalar approach for treatment. 
Figure 1. Transversal (A) and coronal (B) T2-weighted views of a $1 \mathrm{~cm}$ rounded cystic structure, adjacent to the left L5-L6 zygapophyseal joint. This lesion is located anteriorly to the joint and is purely extraforaminal. Neither a foraminal nor intracanalar component can be found.
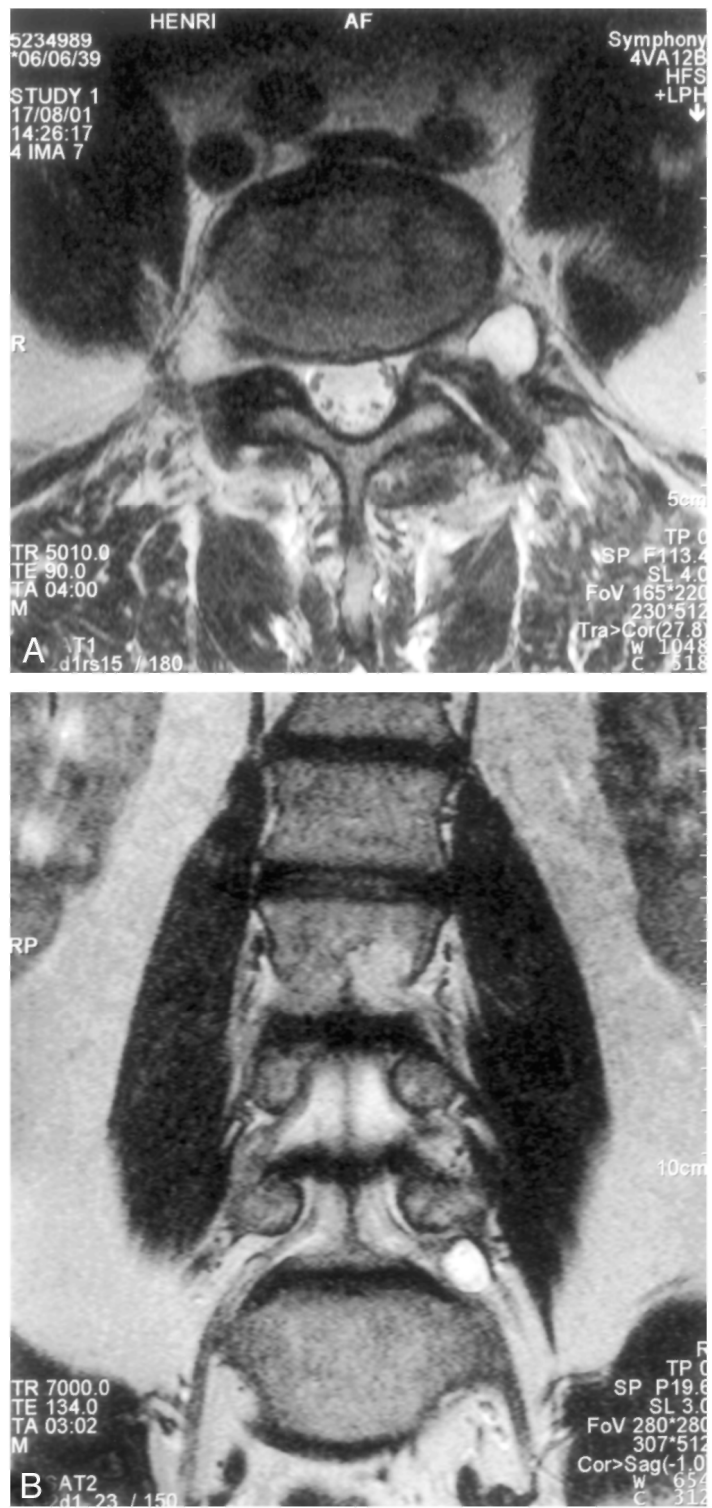

Figure 2. Macroscopical features of the surgical specimen: it is a pseudocyst limited by a thick fibrous wall and containing myxofibrinoid material.

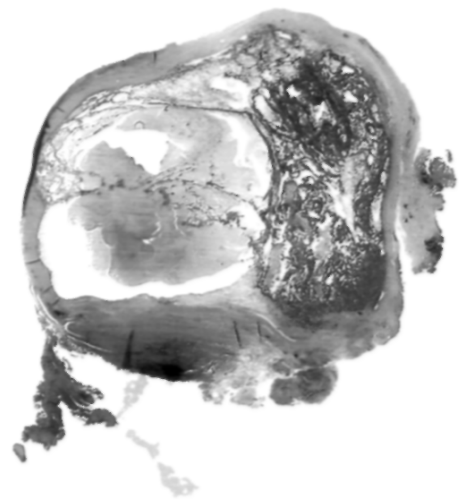


Figure 3. At histology, the cyst wall shows no epithelial or synovial lining and consists of fibrovascular tissue with focal calcium deposits (original magnification x35).

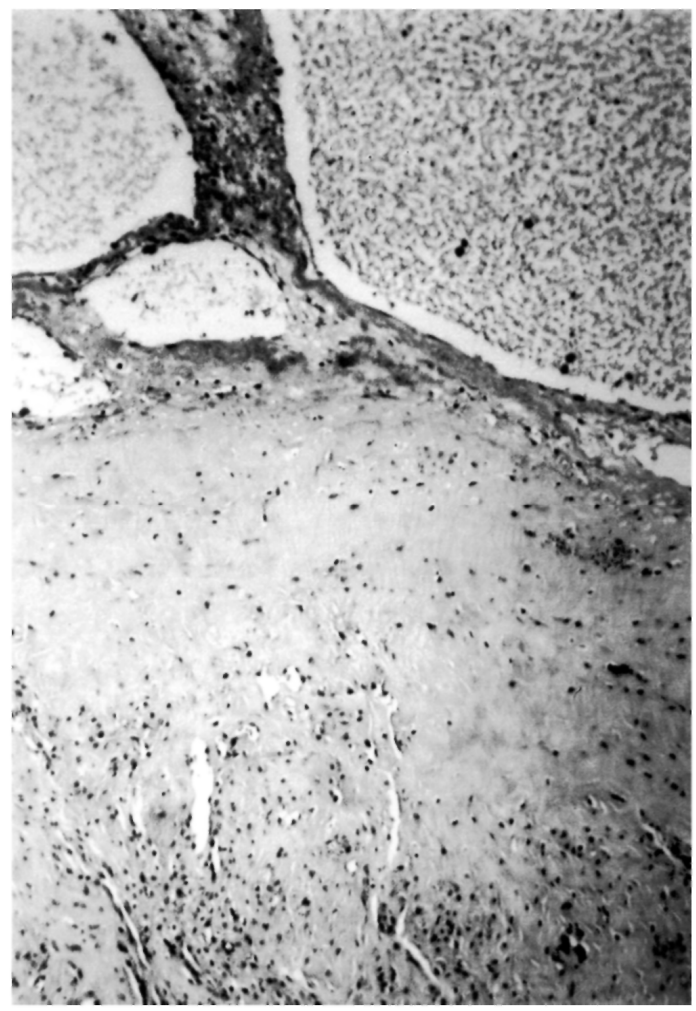

No funds were received in support of this work. No benefits in any form have been or will be received from a commercial party related directly or indirectly to the subject of this manuscript.

\section{References}

1. Kemaloglu S, Nas K, Gur A, et al. Synovial cyst at the intervertebral foramina causing lumbar radiculopathy. Spinal Cord 2001;39:399401.

2. Mercader J, Gomez JM, Cardenal C. Intraspinal synovial cyst: diagnosis by CT. Follow-up and spontaneous remission. Neuroradiology 1985;27: 346-8.

3. Cartwright MJ, Nehls DG, Carrion CA, et al. Synovial cyst of a cervical facet joint: case report. Neurosurgery 1985;16:850-2.

4. Howington JU, Connoly ES, Voorhies RM. Intraspinal synovial cysts: 10-year experience at the Oschner Clinic, J Neurosurg 1999;91:193-9.

5. Lyons MK, Atkinson JR, Wharen RE, et al. Surgical evaluation and treatment of lumbar synovial cysts: the Mayo Clinic experience, $J$ Neurosurg 2000;93(suppl 1):53-7.

6. Sabo RA, Tracy PT, Weinger JM. A series of 60 juxtafacet cysts: clinical presentation, the role of spinal instability, and treatment, $J$ Neurosurg 1996; 85:560-5.

7. Salmon B, Martin D, Lenelle J, et al. Juxtafacet cysts of the lumbar spine. Clinical, radiological and therapeutic aspects in 28 cases. Acta Neurochir (Wien) 2001;143:129-34

8. Pendelton B, Carl B, Pollay M. Spinal extradural benign synovial and ganglion cyst: case report and review of the literature. Neurosurgery 1983;13: 322-6.

9. Haase J. Extradural cyst of ligamentum flavum L4: a case. Acta Orthop Scand 1972;43:32-8.

10. Herrington JL, Edwards LW. Ganglion cysts arising in unusual locations. Ann Surg 1995;142:900-3. 
Published in : Spine (2003), vol. 28, $n^{\circ} 19$, pp. E405-E407

Status : Postprint (Author's version)

11. Kao C, Uihlein A, Bickel W, et al. Lumbar intraspinal extradural ganglion cyst, J Neurosurg 1968;29:168-72.

12. Hatem O, Bedou G, Negre C, et al. Intraspinal cervical degenerative cyst. Report of three cases, J Neurosurg 2001;95(suppl 1):139-42.

13. Yarde WL, Arnold PM, Kepes JJ, et al. Synovial cysts of the lumbar spine: diagnosis, surgical management, and pathogenesis. Report of eight cases. Surg Neurol 1995;43:459-64; discussion 465. 